Ann. Sci. forest., 1977, 34 (1), 59-75.

\title{
Contribution à l'étude de l'échantillonnage en futaie feuillue
}

\author{
J.-P. GRAYET \\ Faculté des Sciences agronomiques de l'Ełat, Chaire de Statistique ef Informatique Gembloux, Belgique
}

\section{Résumé}

L'originalité de notre travail résidant dans la connaissance parfaite d'un important matériel ligneux, tant en ce qui concerne sa localisation que ses dimensions et ses accroissements (résultats complets et détaillés de deux inventaires successifs) ; nous avons abordé l'étude des méthodes d'échantillonnage par la voie de la simulation, en étudiant la précision obtenue par les échantillonnages aléatoire et systématique dans deux types de forêts feuillues ardennaises. De cette manière, nous avons testé différentes surfaces de placettes et fait varier l'intensité d'échantillonnage afin d'examiner l'évolution de la précision des résultats exprimés en nombre de tiges, en surface terrière et en volume ainsi que de leurs accroissements.

En ce qui concerne l'échantillonnage aléatoire, nous pouvons dire que le facteur forme de la parcelle échantillon est très secondaire, et qu'à surface égale, les placettes de diverses formes donneni des précisions pratiquement identiques. Par contre, nous insisterons sur le fait que les résultats obtenus mettent clairement en évidence le rôle prépondérant de l'intensité d'échantillonnage utilisée.

En fonction de l'objectif que l'on s'est fixé et du type de forêt, nous devons définir une limite de précision en dessous de laquelle nous ne voulons pas descendre, ce qui nous amène à donner une idée de la surface minimale nécessaire à inventorier pour obtenir la précision souhaitée en fonction de la variable étudiée (tableau 5).

Nous pouvons grossièrement considérer, que dans la plupart des cas (coef. de variation égal à $5 \%$ ), il faut :

- une superficie minimale de 10 ha pour obtenir des renseignements valables en échantillonnage statique et en échantillonnage dynamique (placettes permanentes et période d'une dizaine d'années) pour la surface terrière et le volume ;

- une superficie minimale de 500 ha pour l'échantillonnage dynamique en placettes temporaires ( $P \sim 10$ ans) des accroissements en surface terrière et en volume.

Ajoutons enfin que la précision obtenue par l'échantillonnage systématique, préféré généralement dans la pratique, n'est guère différente de celle de l'échantillonnage aléatoire, mais que dans l'ensemble, cette technique systématique est la plus précise des deux.

\section{Introduction}

L'intérêt des méthodes d'inventaire par échantillonnage n'étant point mis en doute, nous pensons cependant devoir faire remarquer que, vu la situation complexe et hétérogène de nos forêts, il n'est guère possible de définir des règles générales permettant d'appliquer ces techniques sans discernement. 
L'criginclité c'e notre trcvail résićcrit ócns la ccnnaissance parfaite d'un in:portant matériel ligneux, tant $\in$ ce qui concerne sa localisation que ses dimensions et ses accroissements (résultcts complets et détaillés de deux inventaires successifs), nous nous scmmes citcqués à ce problème por la voie de la simulation, en éfudiant la précision obtenue par les échaníllonnages aléatoire et systématique dans deux types de forêts feuillues ardennaises. De cette manière, nous avons testé différentes surfaces de placettes et fait varier l'intensité d'échantillonnage afin d'examiner l'évolution de la précision des résultats, portant à la fois sur l'évaluation du matériel sur pied et sur l'accroissement de ce matériel. La poursuite de tels objectifs nécessite le traitement d'un très grand nombre de données ; aussi les nombreux calculs ont-ils été réalisés par ordinateur (I.B.M.1130).

Le texte qui suit s'articule en 4 parties complémentaires. La première a pour bu ${ }^{\dagger}$ de définir le contexte général du travail, en donnant un aperçu du milieu naturel, du dispositif expérimental et des mesures effectuées. Dans les deuxième et troisième parties, nous traiterons respectivement de l'échentillonnoge aléctoire et de l'échantillonnage systématique, en examinant pour chacune de ces techniques la méthode d'étude utilisée et les résultats obtenus. Enfin, après avoir pris en considération les travaux réalisés par d'autres auteurs dans ce domaine, nous tenterons, en guise de conclusion, de dégager concrètement l'impact de ces recherches dans la pratique des inventaires partiels.

\section{Le matériel expérimental}

\subsection{La forêt domaniale d'Arilier}

La forêt domaniale d'Anlier fait partie d'un vaste massif forestier s'étendant de la frontière française au Grand-Duché de Luxembourg. A elle seule, la forêt occupe 5487 hectares.

Dans celte forêt, les feuillus l'emportent largement sur les résineux, puisque ceux-ci n'occupent que 5,6 p. 100 de la surface totale. Parmi ces feuillus, nous pouvons dire que la futaie mélangée de chêne et de hêtre domine assez largement. Notons aussi que la rotation y est fixée à douze ans.

Enfin, attirons l'attention sur la présence de caractères particuliers plus ou moins localisés (état du peuplement, proportion de chênes, etc.), directement en rapport avec l'historique de la forêt.

\subsection{Le dispositif expérimental}

L'étude entreprise est basée sur l'existence de parcelles d'expérience installées et déjà inventoriées depuis plus de dix ans. Ce remarquable outil de travail, qui a servi de base à une étude de répartition spatiale des arbres (Lenger, 1961), est l'œuvre de Mme A. Bary-Lenger (1960). C'est à l'amabilité et à la délicatesse de son auteur que nous devons d'avoir pu utiliser les données récoltées.

\subsubsection{Localisation des parcelles d'expérience.}

Les parcelles d'expérience se composent de deux carrés de 16 hectares chacun. La première de ces parcelles est délimitée dans la futaie régulière, alors que la seeunde est située dans une zone d'allure jardinée. 


\subsubsection{Milieu naturel.}

La futaie régulière appartient à deux sous-associations du Luzulo-fagefum, caractérisées entre autres par des sols bruns acides et un humus du type moder.

Dans la futaie jardinée, on distingue diverses associations végétales qui sont directement fonction du relief (variantes du Luzulo-fagefum et Fago-quercetum). Les sols sont plus médiocres que dans la futaie régulière ; l'humus est du type dysmoder.

\subsubsection{Le dispositif expérimental proprement dit.}

Comme nous l'avons déjà dit, nous disposons dans la forêt d'Anlier, de 2 blocs d'expérience distincts, ayant chacun une superficie de 16 hectares (400 mètres $X$ 400 mètres). Chaque parcelle est divisée en 100 sous-parcelles de 16 ares, matérialisées et identifiées sur le terrain au moyen de piquets corniers. A l'intérieur de ces sousunités expérimentales, tous les arbres sont numérotés et portent un repère indiquant la hauteur de mesure de la circonférence.

Chacun des arbres a été localisé sur plan avec précision par ses coordonnées cartésiennes, obtenues lors d'un relevé topographique par triangulation. Les recoupements effectués lors du travail sur le terrain ont conduit à une précision très satisfaisante (Lenger, 1960).

En plus de la mesure des coordonnées, nous possédions au départ une mesure de circonférence pour chacun des arbres. Ces mesures ont été réalisées durant l'été 1960 dans la futaie jardinée et en 1963 dans la futaie régulière.

De notre côté, nous avons refait un inventaire complet des circonférences durant l'hiver 1972-1973, repérant par ailleurs les arbres pris en éclaircie (en 1970-1971 dans la futaie régulière et en 1961-1962 dans la futaie irrégulière). Utilisant la relation obtenue par régression linéaire entre circonférences initiales ef accroissements des arbres présents lors des deux inventaires, nous avons pu estimer les circonférences, au moment de l'éclaircie, des arbres prélevés durant l'intervalle. De plus, chaque tige passée à la futaie (circonférence supérieure ou égale à 50 centimètres) a été localisée et inventoriée.

En conclusion, notre matériel d'étude comporte :

- deux parcelles d'expérience établies dans des structures forestières différentes (régulière et jardinée) ;

- deux inventaires successifs des circonférences (périodes de 9 et 12 ans) ;

- une localisation précise de chaque arbre ;

- la connaissance du passage à la futaie ;

- une estimation du prélèvement obtenue à partir du calcul de circonférences théoriques au moment de l'éclaircie.

\subsubsection{Quelques caractéristiques dendrométriques des parcelles d'expérience.}

Avant d'entamer la partie expérimentale proprement dite, il convient de donner un certain nombre de renseignements quantitatifs obtenus par une $1^{\text {re }}$ exploitation des données brutes (tabl. 1).

Ajoutons que la proportion de chênes reste comprise entre 6 et 7 p. 100 dans la futaie régulière et que les accroissements moyens périodiques en circonférence par individu y sont respectivement de 1,34 centimètres et de 0,71 centimètres pour les 


\section{TABLEAU 1}

Caractéristiques dendrométriques, ramenées à l'ha, des deux parcelles d'expérience

Characteristics (per ha) of both experimental areas

\begin{tabular}{|c|c|c|c|c|c|c|}
\hline & \multicolumn{3}{|c|}{ Futaie régulière } & \multicolumn{3}{|c|}{ Fułaie irrégulière } \\
\hline & $\begin{array}{l}\text { Nombre } \\
\text { de tiges }\end{array}$ & $\begin{array}{c}\text { Surface } \\
\text { terrière } \\
\left(\mathrm{m}^{2}\right)\end{array}$ & $\begin{array}{l}\text { Volume } \\
\left(\mathrm{m}^{3}\right)\end{array}$ & $\begin{array}{c}\text { Nombre } \\
\text { de tiges }\end{array}$ & $\begin{array}{c}\text { Surface } \\
\text { terrière } \\
\left(\mathrm{m}^{2}\right)\end{array}$ & $\begin{array}{c}\text { Volume } \\
\qquad\left(\mathrm{m}^{3}\right)\end{array}$ \\
\hline \multicolumn{7}{|l|}{ 1er inventaire : } \\
\hline Hêtres ...... & 191 & 24,91 & - & 114 & 15,54 & - \\
\hline Chênes $\ldots \ldots \ldots \ldots \ldots$ & 13 & 1,71 & - & 29 & 5,20 & - \\
\hline Total ................. & 204 & 26,62 & 187,98 & 143 & 20,74 & 147,63 \\
\hline \multicolumn{7}{|l|}{$2 \mathrm{e}$ inventaire: } \\
\hline Hêtres ...... & 138 & 22,72 & - & 108 & 16,02 & - \\
\hline Chênes ............. & 9 & 1,47 & - & 23 & 4,90 & - \\
\hline Total .................. & 147 & 24,19 & 171,99 & 131 & 20,92 & 149,58 \\
\hline \multicolumn{7}{|l|}{ Evolution ( 9 et 12 ans) : } \\
\hline Hêtres ............. & -54 & $-2,19$ & - & -6 & $+0,48$ & - \\
\hline Chênes .............. & -4 & $-0,24$ & - & -6 & $-0,30$ & - \\
\hline Total ................... & -57 & $-2,43$ & $-15,99$ & -12 & $+0,18$ & $+1,95$ \\
\hline \multicolumn{7}{|l|}{ Accroissement ( 9 et 12 ans) : } \\
\hline Total .................... & - & $+4,77$ & $+34,90$ & - & $+4,63$ & $+29,20$ \\
\hline
\end{tabular}

hêtres et pour les chênes. Notons enfin que l'accroissement moyen périodique par hectare en surface terrière est de 0,53 mètre carré.

En ce qui concerne la futcie irrégulière, il faut noter une disparition du chêne proportionnellement beaucoup plus forte que celle du hêtre. L'accroissement moyen périodique en circonférence par individu est de 1,47 centimètres pour le hêtre et de 1,05 centimètres pour le chêne. Enfin l'accroissement moyen périodique par hectare en surface terrière atteint 0,39 mètre carré.

\section{Echantillonnage aléatoire}

\subsection{Principes généraux}

Schématiquement, la technique que nous avons utilisée consiste en un découpage fictif des parcelles de 16 hectares en carrés élémentaires d'un are, qui constituent nos plus petites unités d'échantillonnage. Utilisant les techniques du calcul automatique, nous avons procédé à ce découpage et « simulé » divers regroupements de ces carrés élémentaires afin d'obtenir des formes et des surfaces de placettes variables. Nous nous proposons dès lors de calculer les variances pour des placettes de taille croissante et de forme variable. Pratiquement, nous avons pris en considération des placettes ayant au maximum un rapport longueur/largeur égal à 5/2 et des surfaces variant de 4 à 25 ares.

Pour chaque méthode d'inventaire partiel, nous distinguerons l'échantillonnage « statique» de l'échantillonnage « dynamique». 
Par échantillonnage «statique», nous entendons l'étude d'un seul des 2 inventaires. En d'autres termes, notre but est d'estimer la précision obtenue lors de l'évaluation du matériel sur pied à un moment donné.

Par contre, l'échantillonnage « dynamique » est basé sur l'exploitation de 2 inventaires successifs. Nous tenterons en effet de porter un jugement sur la validité des estimations de l'évolution du matériel en un laps de temps plus ou moins long. Nous scinderons les problèmes relatifs à la détermination des accroissements du matériel ligneux en envisageant séparément le cas des placettes permanentes et celui des placettes temporaires.

Afin de fixer les idées, ajoutons que, dans les calculs, nous considérerons les variables suivantes : le nombre de tiges, la surface terrière et le volume.

Parłant des données brutes mesurées en forêt, nous avons mis au point divers programmes et sous-routines de calcul destinés à préparer les données et consistant essentiellement en une totalisation, pour chacune des variables, des valeurs relatives à l'ensemble des arbres situés au sein d'une même placette élémentaire de 1 are.

Dans un premier stade, nous avons localisé chacun des arbres dans un système de coordonnées communes calculées à partir d'une même origine. Chaque arbre a ensuite reçu un numéro-repère indiquant à quel carré élémentaire il appartient. Enfin, nous avons effectué les totalisations, par are et par variable.

\subsection{Précision des inventaires et des comparaisons d'inventaires en placettes permanentes}

\subsubsection{Méthode d'étude.}

Pour chacune des variables, nous avons effectué le calcul des variances ramenées à la parcelle élémentaire de 1 are, pour les parcelles de grandeurs et de formes variables. Le tableau 2, donne un aperçu de la variabilité observée.

Smith (1938) a mis au point un modèle théorique de la variabilité des parcelles d'expérience qui s'exprime comme suit :

$$
\sigma_{\overline{\mathrm{x}}(\mathrm{n})}^{2}=\sigma_{1}^{2} / \mathrm{n}^{\beta}
$$

où : $\sigma_{\overline{\mathbf{x}}(\mathrm{n})}^{2}=$ variance de la moyenne pour des groupes de $\mathrm{n}$ parcelles unitaires ;

$\sigma_{1}^{2}=$ variance des placettes unitaires (placettes de 1 are) ;

$\beta=$ coefficient compris normalement entre 0 et 1 .

En nous inspirant de ce modèle, nous pouvons conclure, dans le cas qui nous occupe, à l'existence de parcelles de 1 are totalement indépendantes lorsque $\beta=1$. Alors que, plus $\beta$ diminue et se rapproche de 0 , plus les parcelles voisines sont corrélées. A la limite pour $\beta=0$, les parcelles sont totalement dépendantes (ou corrélées).

Ignorant les valeurs à attribuer à $\beta$ pour chacune des variables étudiées, nous les avons estimées par régression linéaire pondéréa à partir des variabilités observées. La relation (1) peut en effet s'écrire sous forme logarithmique :

$$
\log \sigma_{\overline{\mathbf{x}}(\mathrm{n})}^{2}=\log \sigma_{1}^{2}-\beta \log \mathrm{n}=\alpha+\beta \log \mathrm{n} .
$$

Cette équation étant de la forme :

$$
y=a+b x,
$$

Annales des Sciences forestières. - 1977. 
TABLEAU 2

Valeurs du coefficient de variation

observé en fonction du type de placette et de la variable étudiée

Observed coefficients of variation for various kinds of plots

and various studied characteristics

\begin{tabular}{|c|c|c|c|c|c|c|c|c|c|c|c|}
\hline \multirow{2}{*}{\multicolumn{3}{|c|}{$\begin{array}{c}\text { Dimensions des pla- } \\
\text { cettes }(\mathrm{m}) \ldots \ldots \ldots \\
\begin{array}{c}\text { Superficies des pla- } \\
\text { cettes(ares) ....... }\end{array}\end{array}$}} & \multirow{2}{*}{$\frac{20 \times 20}{4}$} & \multirow{2}{*}{$\frac{20 \times 40}{8}$} & \multirow{2}{*}{$\frac{40 \times 20}{8}$} & \multirow{2}{*}{$\frac{20 \times 50}{10}$} & \multirow{2}{*}{$\frac{50 \times 20}{10}$} & \multirow{2}{*}{$\frac{40 \times 40}{16}$} & \multirow{2}{*}{$\frac{40 \times 50}{20}$} & \multirow{2}{*}{$\frac{50 \times 40}{20}$} & \multirow{2}{*}{$\begin{array}{r}50 \times 50 \\
25\end{array}$} \\
\hline & & & & & & & & & & & \\
\hline \multirow{3}{*}{ 迹竞 } & \multirow{2}{*}{$\begin{array}{l}\text { Surface } \\
\text { terrière }\end{array}$} & 1er inv... & 24,8 & 17,4 & 16,0 & 14,5 & 15,1 & 11,4 & 10,9 & 10,6 & 9,6 \\
\hline & & $2^{\mathrm{e}}$ inv $\ldots$ & 27,8 & 19,5 & 18,0 & 16,8 & 17,4 & 12,1 & 12,0 & 11,4 & 10,9 \\
\hline & \multicolumn{2}{|c|}{$\begin{array}{c}\text { Accroissement en } \\
\text { surface terrière. }\end{array}$} & 26,4 & 20,1 & 18,8 & 17,9 & 18,0 & 15,2 & 14,6 & 14,7 & 13,5 \\
\hline \multirow{3}{*}{ 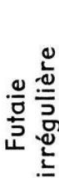 } & \multirow{2}{*}{$\begin{array}{l}\text { Surface } \\
\text { terrière }\end{array}$} & $1 \mathrm{er}$ inv.. & 37,2 & 26,2 & 26,6 & 22,8 & 24,0 & 19,8 & 16,3 & 17,1 & 12,3 \\
\hline & & $2^{e}$ inv... & 39,0 & 28,0 & 26,9 & 24,8 & 23,2 & 19,6 & 16,7 & 17,2 & 12,3 \\
\hline & \multicolumn{2}{|c|}{$\begin{array}{c}\text { Accroissement en } \\
\text { surface terrière.. }\end{array}$} & 34,7 & 25,5 & 25,8 & 24,1 & 23,2 & 19,5 & 18,2 & 17,5 & 16,3 \\
\hline
\end{tabular}

en reprenant les valeurs observées de $\sigma_{\overline{\mathbf{x}}(\mathrm{n})}^{2}$ et celles de $\mathrm{n}$, nous pouvons obtenir $\widehat{\beta}$ et $\log \hat{\sigma}_{1}^{2}$ ou $\hat{\alpha}$ (ordonnée à l'origine), en considérant la relation comme linéaire dans les limites qui nous intéressent.

Compte tenu de toutes ces considérations, nous avons pu mettre en évidence les équations suivantes * :

- futaie régulière :

nombre de tiges

surface terrière

volume

évolution du nombre de tiges

accroissement en surface terrière

accroissement en volume

- futaie irrégulière :

nombre de tiges

surface terrière

volume

évolution du nombre de tiges

$\log \sigma_{\overline{\mathbf{x}}(\mathrm{n})}^{2}=-0,1905-0,6764 \log \mathrm{n}$

$\log \sigma_{\bar{x}(n)}^{2}=6,2859-1,0741 \log n$

$\log \sigma_{\bar{x}(\mathrm{n})}^{2}=5,9958-1,0782 \log n$

$\log \sigma_{\bar{x}(\mathrm{n})}^{2}=-0,4604-0,8714 \log n$

$\log \sigma_{\frac{x}{x}(n)}^{2}=4,6342-0,7507 \log n$

$\log \sigma_{\overline{\mathbf{x}}(\mathrm{n})}^{2}=4,3687-0,7645 \log n$

accroissement en surface terrière

accroissement en volume

$\log \sigma_{\overline{\mathrm{x}}(\mathrm{n})}^{2}=-0,0809-0,7519 \log \mathrm{n}$

$\log \sigma_{\bar{x}(\mathrm{n})}^{2}=6,4568-1,0619 \log n$

$\log \sigma_{\overline{\mathrm{x}}(\mathrm{n})}^{2}=6,1748-1,0576 \log \mathrm{n}$

$\log \sigma_{\bar{x}(n)}^{2}=-0,0628-0,9319 \log n$

$\log \sigma_{\overline{\mathrm{x}}(\mathrm{n})}^{2}=4,9144-0,8238 \log \mathrm{n}$

$\log \sigma_{\bar{x}(\mathrm{n})}^{2}=4,6552-0,9147 \log n$

* Tous les logarithmes utilisés sont des logarithmes décimaux. 
En relation avec le tableau 2, nous donnons les valeurs du coefficient de variation estimé à partir des relations précédentes, pour diverses variables et superficies de placettes (tabl. 3). Remarquons que, dans ces deux tableaux, nous nous sommes limités à donner les résultats qui concernent la surface terrière, tant sont semblables caux obtenus pour le volume.

\section{TABLEAU 3}

Valeurs du coefficient de variation estimé en fonction du type de placette et de la variable étudiée Estimated coefficients of variation for various kinds of plots and various studied characteristics

\begin{tabular}{|c|c|c|c|c|c|c|c|}
\hline & $\begin{array}{l}\text { Superficies des placettes } \\
\text { (ares) }\end{array}$ & 4 & 5 & 10 & 16 & 20 & 25 \\
\hline \multirow{2}{*}{$\begin{array}{l}\text { Futaie } \\
\text { régulière }\end{array}$} & Surface terrière........... & 26,0 & 23,1 & 15,9 & 12,8 & 11,0 & 9,7 \\
\hline & $\begin{array}{l}\text { Accroissement en surface ter- } \\
\quad \text { rière } \ldots \ldots \ldots \ldots \ldots \ldots \ldots \ldots\end{array}$ & 25,9 & 23,8 & 18,3 & 15,7 & 14,1 & 13,0 \\
\hline \multirow{2}{*}{$\begin{array}{l}\text { Futaie } \\
\text { irrégulière }\end{array}$} & Surface terrière............ & 38,6 & 34,2 & 23,7 & 19,1 & 16,4 & 14,6 \\
\hline & $\begin{array}{l}\text { Accroissement en surface ter- } \\
\text { rière } \ldots \ldots \ldots \ldots \ldots \ldots \ldots \ldots\end{array}$ & 34,5 & 31,4 & 23,6 & 20,0 & 17,8 & 16,2 \\
\hline
\end{tabular}

\subsubsection{Calcul de la précision.}

A partir des équations moyennes du type (2), obtenues pour chaque variable, nous pouvons déterminer les différentes valeurs de $\widehat{\sigma}_{\overline{\mathrm{x}}(\mathrm{n})}$ pour des grandeurs de placettes variant de 4 à 25 ares.

La variance attendue d'un échantillon de $n$ ares est égale à :

$$
\hat{\sigma}_{\text {éch }}^{2}=\hat{\sigma}_{\bar{x}(n)}^{2}(1-f) / p
$$

si $(1-f)$ est le facteur correctif intervenant dans le cas d'une population finie et si $p$ est l'effectif de l'échantillon.

D'autre par $p$ étant égal à :

$$
\frac{100 \mathrm{sf}}{\mathrm{n}}
$$

si s est la surface totale de la population à laquelle on s'intéresse et $f$ la fraction sondée, on $a$ :

$$
\hat{\sigma}_{\text {éch }}^{2}=\frac{\hat{\sigma}_{1}^{2}(1-f)}{n^{\hat{\beta}}} \frac{n}{100 s f}=\frac{\widehat{\alpha} n^{1-\hat{\beta}}(1-f)}{100 s f}
$$

ou sous forme logarithmique :

$$
\log \hat{\sigma}_{\text {êch }}^{2}=\log \hat{\alpha}+(1-\widehat{\beta}) \log n-\log 100-\log s+\log (1-f)-\log f .
$$

Le coefficient de variation s'exprimera comme suit :

$$
\hat{V}_{e c h}=\frac{10}{n} \sqrt{\frac{\hat{\alpha n^{1-\hat{\beta}}(1-f)}}{s f}} .
$$




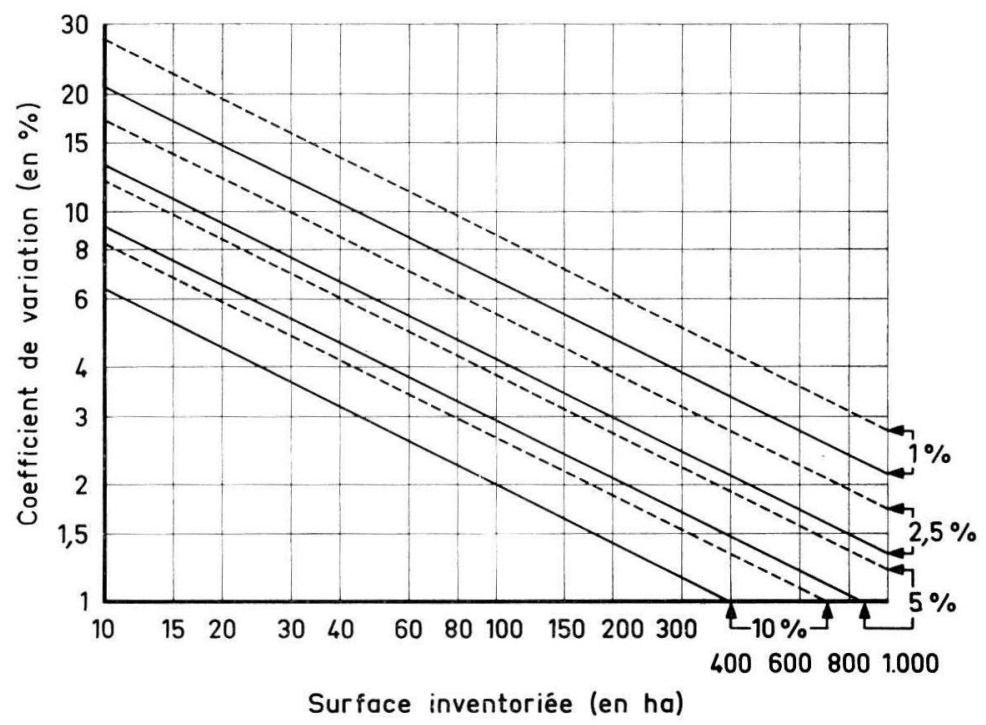

ABAQUE 1. - Nombre de tiges.

Traits continus : futaie régulière.

Traits discontinus : futale irrégulière.

FIG. 1. - Number of trees.

Full line : even-aged stand.

Broken line : uneven-aged stand.

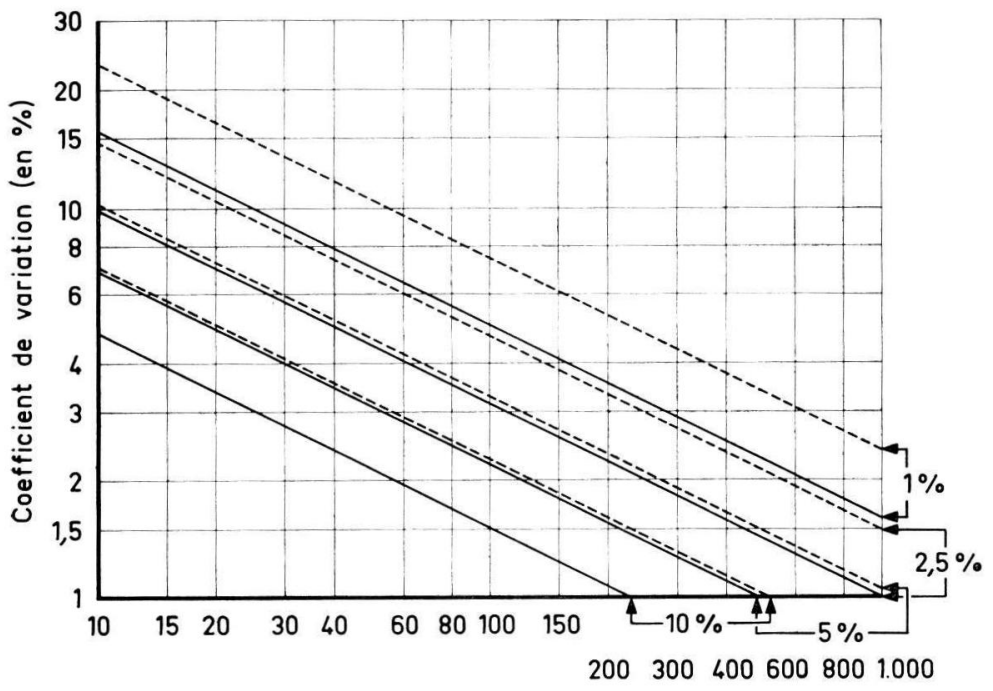

Surface inventoriée (en ha)

Abaque 2. - Surface terrière.

Traits continus : futaie régulière.

Traits discontinus : futaie irrégulière.

FIG. 2. - Basal area.

Full line : even-aged stand.

Broken line : uneven-aged stand. 


\subsubsection{Résultats obtenus.}

Suivant la relation (7), nous pouvons calculer les coefficients de variation $\left(\widehat{V}_{\text {éch }}\right)$ pour différentes valeurs de $n$, de $f$ et de $s$, et ceci pour chacune des 4 variables suivantes : le nombre de tiges, la surface terrière (qui donne des résultats tout à fait identiques à ceux obtenus pour le volume), l'évolution du nombre de tiges et l'accroissement en surface terrière (ou en volume), en futaie régulière et irrégulière.

Pour chacune de ces variables, nous avons dressé un abaque (abaques 1 à 4), où sont représentés en abscisses les différentes valeurs de $s$, allant de 10 à 1000 hectares, et en ordonnées les diverses valeurs obtenues pour le coefficient de variation.

Puisqu'il n'existe que peu de différences, au point de vue précision, pour des placettes de grandeurs différentes (pratiquement, dans le cas de la surface terrière et du volume, « $n$ » n'a pas d'influence), nous n'avons reporté graphiquement, par souci de clarté, que les droites relatives à des placettes de 5 ares.

Simultanément, nous avons fait varier la fraction sondée, considérant successivement des valeurs de fégales à 1 p. 100, 2,5 p. 100, 5 p. 100 et 10 p. 100 . Dans chaque abaque, apparaissent donc 4 paires de droites, correspondant pour les 4 valeurs de $f$ aux limites d'un ensemble de valeurs de $V$ définies en fonction de la structure de la forêt.

Si nous voulons expliciter ces résultats, nous pouvons dire, en assimilant la population à une population normale, que dans environ 2 tiers des cas, la moyenne vraie de la population $(\mathrm{m})$ est contenue dans un intervalle symétrique par rapport à la moyenne

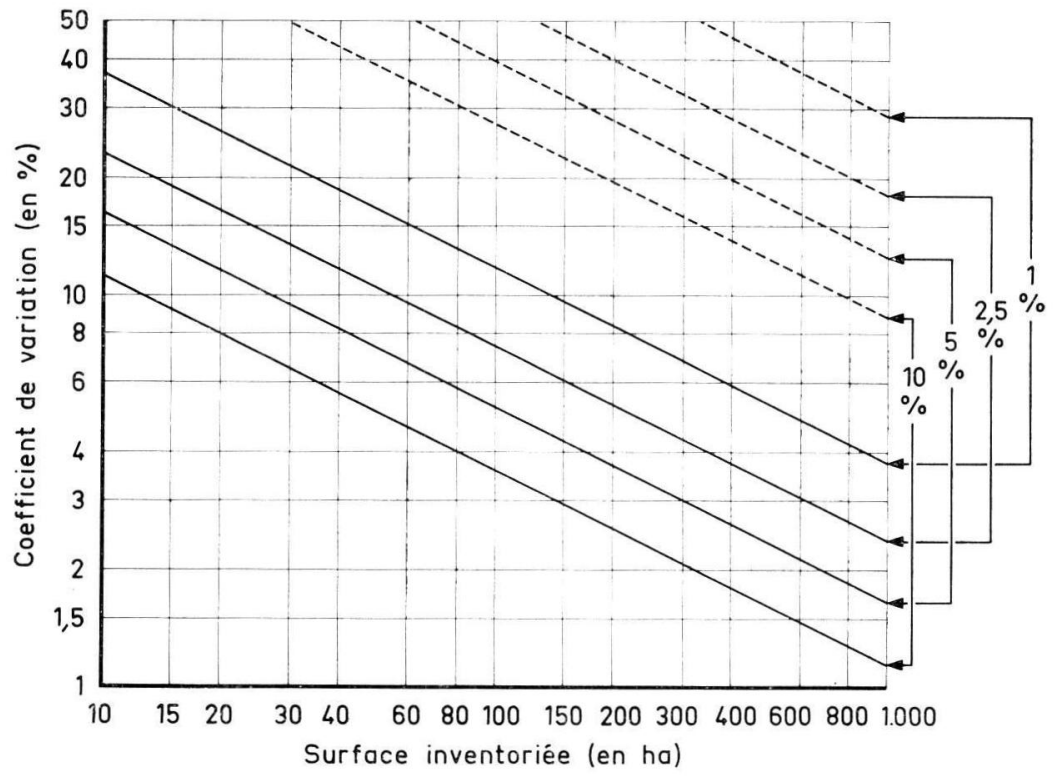

ABAQUE 3. - Evolution du nombre de tiges (placettes permanentes).

Traits continus : futaie régulière.

Traits discontinus : futaie irrégulière.

FIG. 3. - Evolution of the number of trees (permanent plots).

Full line : even-aged stand.

Broken line : uneven-aged stand. 


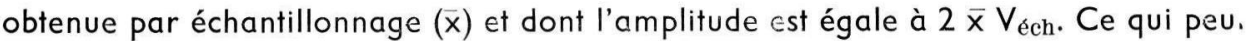
encore s'écrire :

$$
\hat{\mathrm{m}}=\overline{\mathrm{x}} \pm \overline{\mathrm{x}} \mathrm{V}_{\text {éch. }}
$$

De même pour des degrés de confiance de 95 et 99 p. 100, nous aurons respectivement :

et

$$
\widehat{\mathrm{m}}=\overline{\mathrm{x}} \pm 2 \times \mathrm{V}_{\text {éch }} \text {. }
$$

$$
\widehat{\mathrm{m}}=\overline{\mathrm{x}} \pm 2,6 \overline{\mathrm{x}} \mathrm{V}_{\text {éch }} \text {. }
$$

Admettant qu'il existe peu de différences dans les valeurs de $V_{\text {éch }}$ pour des placettes de grandeurs différentes, nous ajouterons cependant que la structure de la forêt influe très sensiblement sur les valeurs du coefficient de variation, celles-ci étant plus faibles dans une forêt homogène (futaie régulièrє) que dans une forêt où le matériel est plus hétérogène (futaie irrégulière), quelle que soit la variable étudiée.

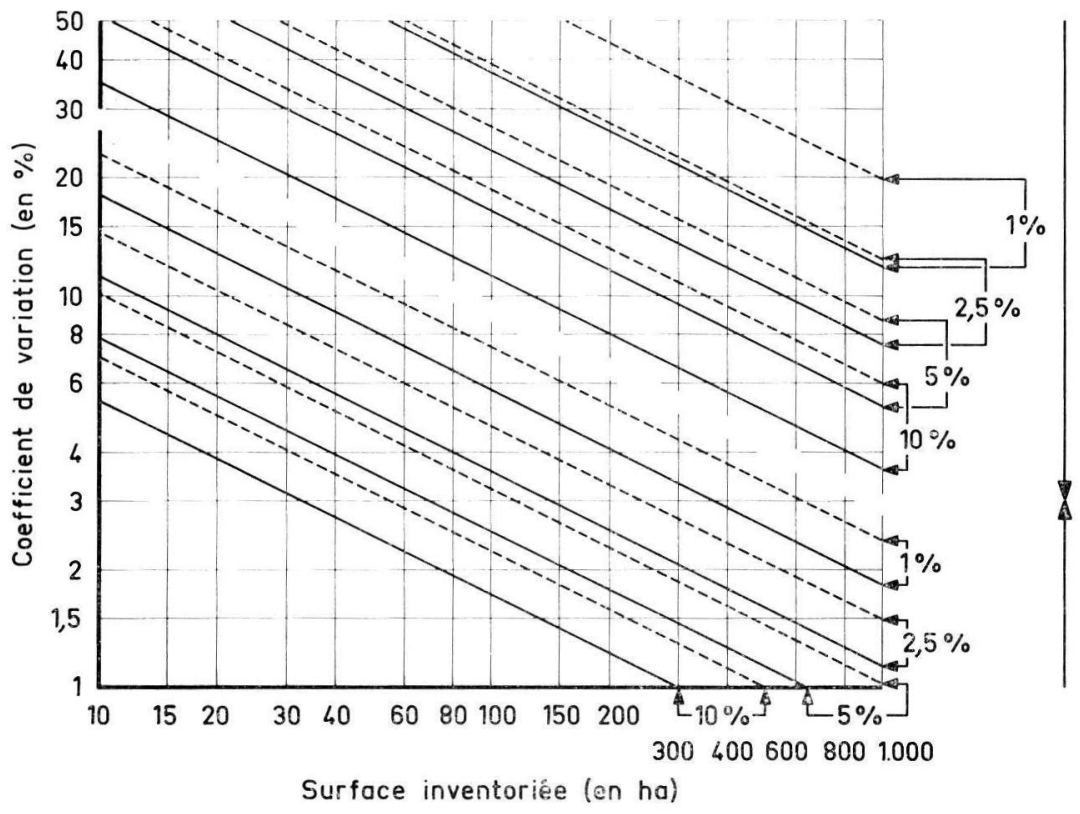

ABAQUE 5.

ABAQUE 4

ABAQUE 4. - Accroissement en surface terrière (placettes permanentes).

Traits continus : futaie régulière.

Traits discontinus : futaie irrégulière.

FIG. 4. - Basal area increment (permanent plots).

Full line : even-aged stand.

Broken line: uneven-aged stand.

ABAQUE 5. - Accroissement en surface terrière (placettes temporaires, période de 9 ans).

Traits continus : futaie régulière.

Traits discontinus : futaie irrégulière.

FIG. 5. - Basal area increment (temporary plots, 9 year period).

Full line : even-aged stand.

Broken line : uneven-aged stand. 
Nous admettrons pourtant, au vu des résultats, que c'est avant tout le facteur fraction sondée qui conditionne la précision des résultats obtenus.

\subsection{Précision des comparaisons d'inventaires en placettes temporaires}

\subsubsection{Approche théorique.}

Considérant l'accroissement non plus comme une variable propre, mais comme la différence de deux variables indépendantes (valeurs du matériel aux premier et deuxième inventaires), à laquelle nous devons ajouter le matériel pris en éclaircie, la variance relative à l'accroissement s'écrira :

$$
\sigma_{\mathrm{i}}^{2}=\sigma_{\mathrm{inv} .1}^{2}+\sigma_{\mathrm{inv.2}}^{2}+\sigma_{\mathrm{écl}}^{2}
$$

car :

$$
\sigma_{\mathrm{X} \pm \mathrm{Y} \pm \mathrm{Z}}^{2}=\sigma_{\mathrm{X}}^{2}+\sigma_{\mathrm{Y}}^{2}+\sigma_{\mathrm{Z}}^{2}
$$

si $X, Y$ et $Z$ sont trois variables slochastiquement indépendantes.

Admettant que les éclaircies sont connues sans erreur notable, nous pouvons supprimer le 3 e terme de la relation (8).

Enfin, si nous passons au coefficient de variation, nous aurons :

$$
V_{i}=\frac{\sqrt{\sigma_{i}^{2}}}{i}=\frac{\sqrt{\sigma_{\text {inv. } 1}^{2}+\sigma_{\text {inv. } 2}^{2}}}{i}
$$

si $\bar{i}$ est l'accroissement périodique du matériel.

\subsubsection{Résultats obtenus.}

La présence de $\bar{i}$ au dénominateur de l'expression donnant $V_{i}$ montre clairement qu'une réduction de la période considérée détermine automatiquement une hausse sensible des valeurs du coefficient de variation.

Afin de pouvoir comparer les résultats relatıfs aux deux parcelles, dont les calculs d'accroissements portent respectivement sur des périodes de 9 et 12 ans, nous avons dressé des abaques (abaques 5 et 6) pour une période standard de 9 ans. Pour réaliser ces graphiques, nous avons considéré que la valeur de l'accroissement $\bar{i}$ était proportionnelle à la durée de la période. Biologiquement cette hypothèse est sans doute discutable, mais elle nous permet pourtant de comparer les résultats des 2 parcelles.

Sans qu'il soit besoin de reprendre une à une les variables testées, nous pouvons nous demander, compte tenu de l'ampleur des valeurs obtenues pour $V_{i}$, si l'échantillonnage en placettes temporaires possède encore réellement un sens, et si dans les conditions habituellement rencontrées dans nos régions, l'interprétation de tels résultats ne prête pas à critique très sévère (l'explicitation des résultats à partir de $V_{i}$ se faisant également comme mentionné en 3.2.3.). En effet, quel crédit peut-on encore accorder aux accroissements obtenus par des méthodes dont la précision n'est qu'illusoire, surtout si l'on songe que ces résultats doivent guider notre sylviculture?

Nous pourrions peut-être admettre, mais toujours avec les restrictions qui s'imposent et l'obtention inévitable de résultats fort peu précis, l'utilisation d'une telle technique pour des surfaces très vastes et des périodes fort longues. 


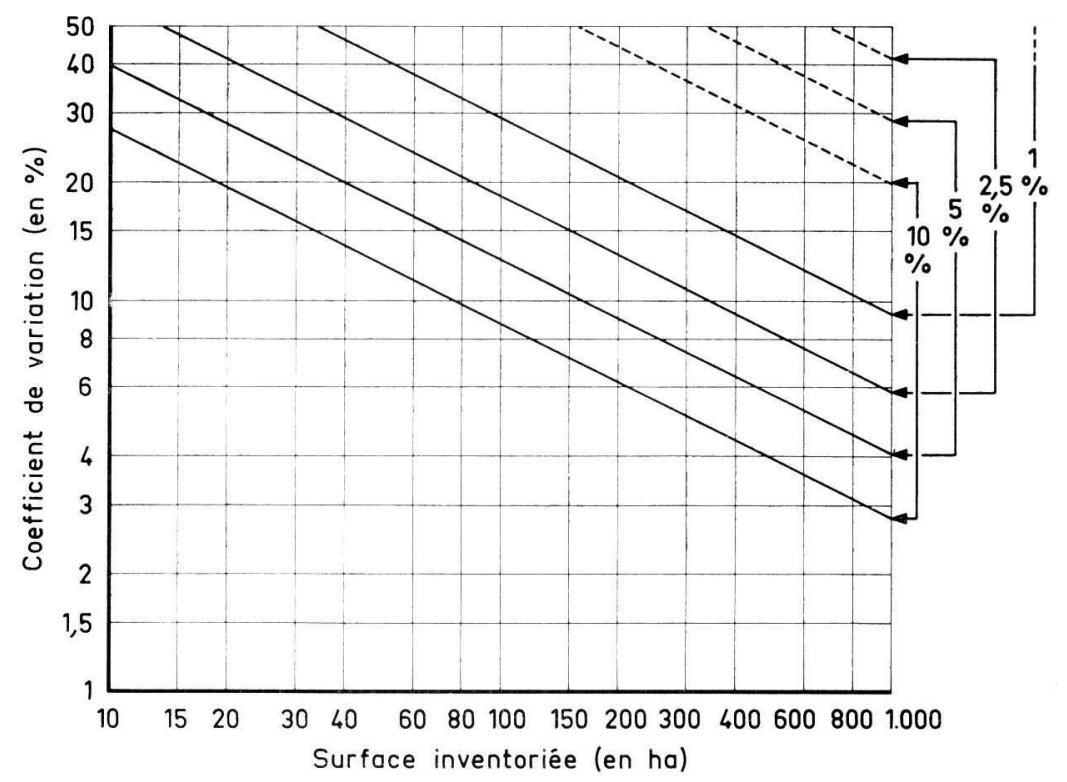

ABAQUE 6. - Evolution du nombre de tiges (placettes temporaires, période de 9 ans).

Traits continus : futaie régulière.

Traits discontinus : futaie irrégulière.

FIG. 6. - Evolution of the number of trees (temporary plots, 9 year period).

Full line : even-aged stand.

Broken line : uneven-aged stand.

\section{Echantillonnage systématique}

\subsection{Principe de calcul et efficacité relative}

Partant de la parcelle carrée de 1600 ares, nous lui avons superposé divers réticules, afin de simuler plusieurs types d'échantillonnage systématique différant les uns des autres par la grandeur des placettes, par la fraction sondée et donc aussi par la forme des mailles.

Pour chaque type de placettes et pour chacun des réticules correspondant, nous avons effectué un maximum de répétitions (le nombre de répétition est égal au rapport de la surface de la maille à celle de la placette). Ces répétitions consistent en fait en une modification de l'emplacement de la parcelle-échantillon de départ.

Après avoir calculé la variance entre répétitions pour une variable, une structure et un type d'échantillonnage donnés, nous avons procédé à un changement d'unité afin d'obtenir les variances unitaires, c'est-à-dire les variances propres aux placettes de 1 are. Celles-ci ont été obtenues en appliquant la relation suivante :

$$
\sigma_{1}^{2}=b^{2} \sigma_{1 / b}^{2}
$$

où $1 / b$ est la surface des placettes d'expérience ; car si :

$$
x^{\prime}=a+b x,
$$


on a :

$$
\sigma_{\mathrm{x}^{\prime}}^{2}=\mathrm{b}^{2} \sigma_{\mathrm{x}}^{2}
$$

Possédant des résultats forts complets pour l'échantillonnage aléatoire, nous avons envisagé l'échantillonnage systématique sous l'angle de la comparaison avec celui-ci.

Nous adopterons la définition donnée par Yates (1949) en ce qui concerne la précision relative (ou efficacité relative) de 2 méthodes différentes d'échantillonnage, basées sur le même type d'unité de sondage. Elle s'exprimera donc comme « l'inverse du rapport des variances d'échantillonnage des estimations données par les deux méthodes lorsqu'on prélève le même nombre d'unités ».

\section{TABLEAU 4}

Efficacité de l'échantillonnage systématique par rapport à la méthode de prélèvement au hasard (en p. 100)

Relative efficiency of systematic and random samplings (in p. 100)

\begin{tabular}{|c|c|c|c|c|}
\hline & \multirow{2}{*}{$\begin{array}{l}\text { Fractions sondées (p. 100) } \ldots \ldots \ldots \ldots \ldots \ldots \ldots \\
\text { Superficies des placettes (ares) } \ldots \ldots \ldots \ldots \ldots \ldots\end{array}$} & \multirow{2}{*}{$\begin{array}{l}1 \\
4\end{array}$} & \multicolumn{2}{|c|}{4} \\
\hline & & & 4 & 16 \\
\hline $\begin{array}{l}\text { Futaie } \\
\text { régulière }\end{array}$ & 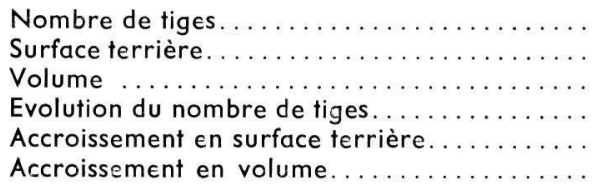 & $\begin{array}{r}91,5 \\
97,5 \\
98,2 \\
100,8 \\
81,1 \\
81,2\end{array}$ & $\begin{array}{r}70,1 \\
77,7 \\
78,8 \\
104,7 \\
61,5 \\
62,0\end{array}$ & $\begin{array}{r}93,5 \\
126,4 \\
129,0 \\
134,3 \\
91,8 \\
92,6\end{array}$ \\
\hline $\begin{array}{c}\text { Futaie } \\
\text { irrégulière }\end{array}$ & 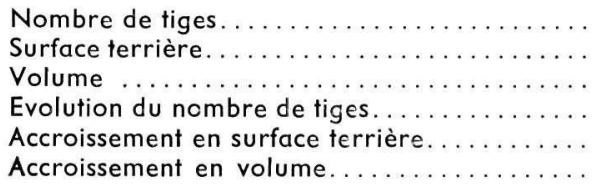 & $\begin{array}{r}122,2 \\
119,9 \\
119,3 \\
107,0 \\
98,4 \\
119,4\end{array}$ & $\begin{array}{l}132,5 \\
120,0 \\
117,3 \\
194,6 \\
136,3 \\
123,0\end{array}$ & $\begin{array}{l}157,5 \\
101,2 \\
101,4 \\
107,6 \\
113,4 \\
114,6\end{array}$ \\
\hline
\end{tabular}

\section{2 Résultats obtenus}

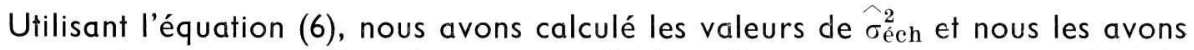
mises en relation avec celles obtenues par l'échantillonnage systématique. Afin de pouvoir confronter les résultats, nous avons dû nous limiter à certaines valeurs particulières de $n$ et de $f$.

Les résultats que nous présentons au tableau 4, traduisent l'efficacité de l'échantillonnage systématique par rapport à la technique aléatoire. Cette efficacité relative est obtenue par la relation :

$$
E_{\mathrm{r}}(\mathrm{p} .100)=100 \frac{\widehat{\sigma}_{\text {alea. }}^{2}}{\widehat{\sigma}_{\text {syst. }}^{2}} .
$$


Elle est directement liée à l'hétérogénéité du matériel inventorié ( $E_{\mathrm{r}}$ est de l'ordre de 100 p. 100 pour la futaie régulière et est compris entre 100 et 200 p. 100 dans la futaie irrégulière). Ainsi, dans une futaie d'allure jardinée, l'échantillonnage systématique se montre supérieur, par suite d'une répartition régulière des placettes permettant de mieux tenir compte des nombreuses variations locales.

En fait cela ne doit guère nous surprendre : l'échantillonnage systématique n'es ${ }^{\dagger}$ somme toute rien d'autre qu'un échantillonnage stratifié dont la stratification serait poussée à l'extrême.

\section{Discussion et conclusions}

L'étude des techniques de l'échantillonnage par les méthodes du calcul rapide (ordinateur) sont relativement récentes : nous citerons à ce sujet O'Regan ef Palley (1965). Quant à la technique de simulation de formes et de grandeurs de placettes, basée sur le regroupement à partir de placettes élémentaires, elle a déjà été utilisée par Johnson et Hixon en 1952 et par Villeneuve en 1964. Le Finlandais Nyyssönen reprend ce principe lors de l'étude comparative des échantillonnages aléatoire et systématique, en 1971. Les dispositifs expérimentaux et les techniques de regroupement précités ont retenu notre attention tant ils sont proches de ceux qui nous ont permis de mener à bien le présent travail.

En ce qui concerne l'échanîillonnage aléatoire, sans vouloir affirmer que la forme des placettes ne joue absolument aucun rôle dans la précision de l'inventaire, nous pouvons dire comme Kulow (1966) que le facteur forme de la parcelle échantilIon est très secondaire, et qu'à surface égale, les placettes de diverses formes donnent des précisions pratiquement identiques. De même, sans négliger les recherches entreprises sur la détermination de l'aire optimale des placeites par Fresse (1961), Tardif (1965) et Nyyssönen et al. (1971), nous insistons sur le fait que les résultats que nous avons obtenus mettent clairement en évidence le rôle prépondérant de l'intensité d'échantillonnage utilisée. Il est indéniable que c'est avant tout le facteur fraction sondée qui conditionne la précision des résultats obtenus, quelle que soit la dimension des placettes.

Mais avant de pouvoir opter pour une intensité et une grandeur de placette, nous devons logiquement nous fixer une limite de précision en dessous de laquelle nous ne voulons pas descendre. Celle-ci est, entre autres choses, fonction de l'objectif que l'on se fixe et du type de forêt. Parde (1961) fixe conventionnellement les limites inférieures de précision ( 2 fois le coefficient de variation) à 5 p. 100 pour les forêts de valeur exceptionnelle, à 7 p. 100 pour les forêts classiques, à 10 p. 100 pour les peuplements médiocres et à 15 p. 100 pour les estimations rapides. De même, nous caractériserons les différents seuils de précision à adopter suivant les cas par les valeurs 2,5 p. 100,5 p. 100,10 p. 100 et 20 p. 100 du coefficient de variation. Ceci nous amène à donner une idée de la surface minimale nécessaire à inventorier pour obtenir ces diverses précisions en foncíion de la variable étudiée. Le tableau 5 nous donne l'ordre de grandeur de ces superficies, pour une intensité d'échantillonnage de 10 p. 100 et pour la grandeur de placette la plus favorable, ce qui, dans le cas de placettes de 4 ou 5 ares, donnerait 2 placettes par hectare. La plupart des cellules du 
TABLEAU 5

Ordre de grandeur des superficies minimales à inventorier (suivant l'hétérogénéité de la forêt) pour des parcelles de grandeur optimale et pour une intensité d'échantillonnage de 10 p. 100 *

Minimals areas to inventory (according to the heterogeneity of the forest) for various optimum sizes of plots and for a sampling fraction of 10 p. 100

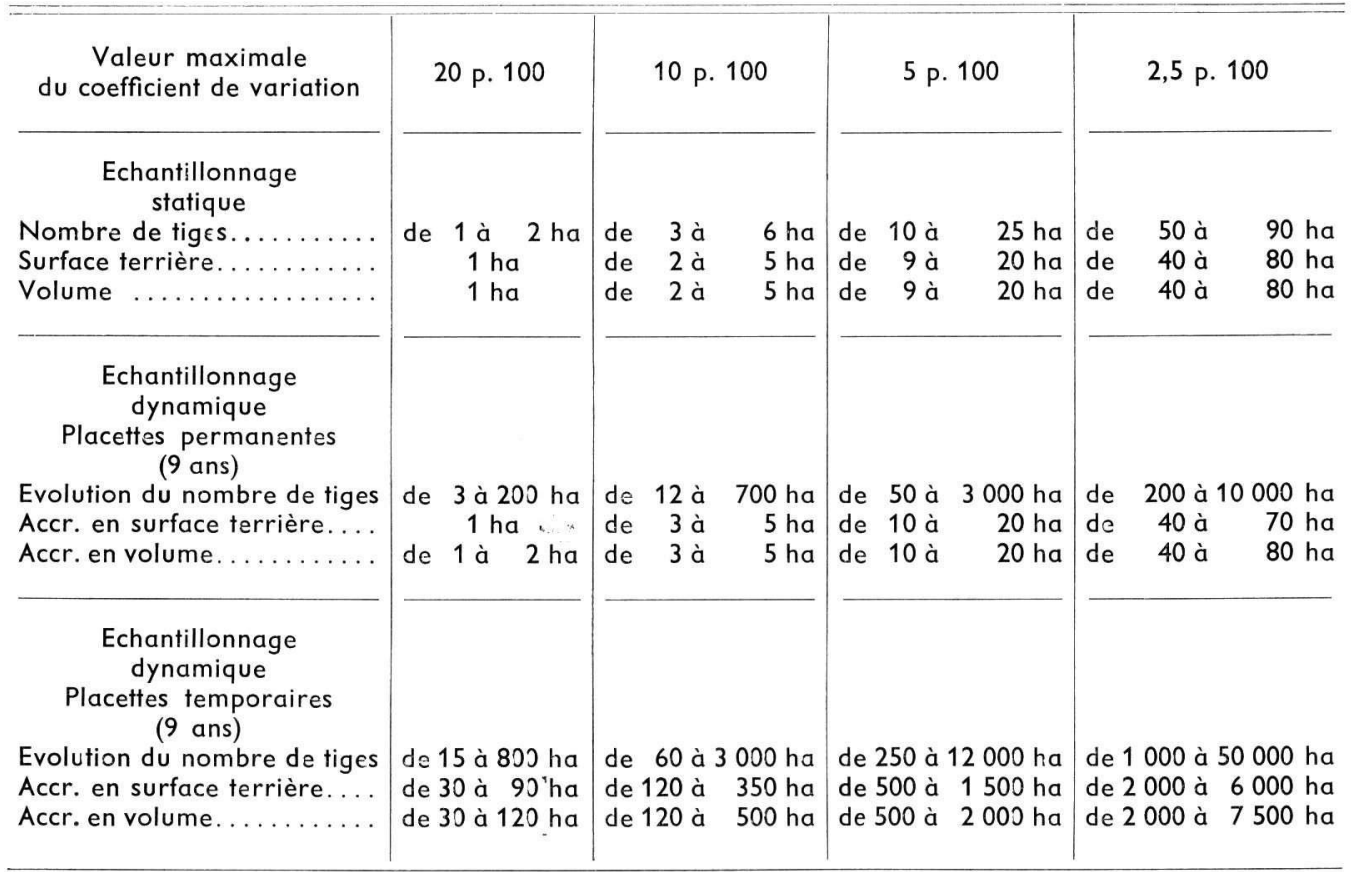

* Les limites inférieures et supérieures correspondent respectivement à des milieux homogène (futaie régulière) et hétérogène (fulaie irrégulière).

tableau contiennent 2 chiffres, qui sont les limites attendues correspondant respectivement à une forêt homogène et à une forêt hétérogène.

Nous pouvons grossièrement considérer que, dans la plupart des cas (coefficient de variation égal à 5 p. 100), il faut :

- une superficie minimale de 10 hectares pour obtenir des renseignements valables en échantillonnage statique et en échantillonnage dynamique (placettes permanentes et période d'une dizaine d'années) pour la surface terrière et le volume. Ce qui ne signifie pas pour autant qu'il faille dans ces conditions pratiquer automatiquement l'inventaire par échantillonnage sur des surfaces de l'ordre de 10 hectares. Les résultats pour l'évolution du nombre de tiges étant nettement moins précis, il est clair qu'il serait vain dans ces conditions de vouloir obtenir des données valables sur des modifications des disíributions des tiges par catégories de grosseur ;

- une superficie minimale de 500 hectares pour l'échantillonnage dynamique en placettes temporaires (pour une période d'une dizaine d'années) pour les accroissements en surface terrière et en volume. Loetsch et Haller (1966) sont eux aussi sceptiques quant à l'intérêt des placettes temporaires pour des calculs d'accroissements. 
Quant à l'échantillonnage systématique, préféré généralement dans la pratique, nous pouvons, au vue de nos résultats, nous rallier à l'avis de nombreux auteurs, tels Hasel (1938), Parde (1956), Nyyssönen et al. (1971), et même à celui plus nuancé de Kulow (1966), pour dire que les précisions obtenues par les échantillonnages systématique et aléatoire ne sont pas très différentes et que, dans l'ensemble, la technique systématique est la plus précise des deux. Adopter les normes définies au tableau 5 pour un inventaire partiel systématique ne peut conduire, dans la plupart des cas, qu'à des résultats dont la précision sera supérieure à celle escomptée.

Nous conclurons en disant que, en possession de ces renseignements concernant la précision, nous pensons pouvoir guider le praticien en lui indiquant les méthodes compatibles avec les moyens dont il dispose et avec les objectifs qu'il s'est fixés.

\title{
Envoyé par l'auteur pour publication en janvier 1975.
}

\author{
Summary \\ Contribution to the study \\ of sampling methods in deciduous forests
}

\begin{abstract}
Using an extensive source of information (complete and detailled results from two successive inventories) on an important stock of trees (important for its position, vastness and rate of growth), we study the precision obtained by systematic and random sampling from two kinds of deciduous forests situated in the Ardennes by computer simulation methods. We tested various sizes of plots and varied the sampling intensity in order to examine the sensitivity of the observed volumes, basal areas and number of trees and their increments.

it appears that for random sampling the shape of the plot is only of secundary importance and that plots of different shapes but equal area yield almost identical precisions. On the other hand the results show clearly the primary importance of the sampling intensity.

A critical lower bound for the precision of our measurements should be chosen taking account of the type of forest and the objective of the study. Table 5 gives for different variables the minimal area to be inventorised in order to attain the critical lower bound for the precision.

We find that in most cases (coefficient of variation of 5 p. 100) we need :

a) Minimal area of 10 ha when using static sampling and dynamic sampling (permanent plots and a time period of 10 years) for the basal area and the volume.

b) Minimal area of 500 ha for dynamic sampling from temporary plots (period of 10 years) for the basal area and the volume increments.

The precisions of the results obtained from random sampling and the more widely used systematic sampling yield results of comparable precision. On the whole, systematic sampling is the more precise method.
\end{abstract}

\section{Références bibliographiques}

FREESE F., 1961. Relation of plot size to variability : an approximation. J. For., 61 (59), 679.

HASEL A. A., 1938. Sampling error in timber surveys. J. Agr. Res., 52, 713-736.

JOHNSON A., HIXON J., 1952. The most efficient size and shape of plot to use for cruising in old growth Douglas - fir timber. J. For., 50, 17-20.

KULOW D. L., 1966. Comparaison of forest sampling designs. J. For., 64 (7), 469-474.

LENGER A., 1960. Les méthodes d'échantillonnage en sylviculture. Bull. Inst. Agron. Stn. Rech. Gerrbloux, H. S., 1, 318-330.

LENGER A., 1961. Etude de la répartition des arbres dans une futaie feuillue. Paris, $33^{e}$ Session. Bull. Inst. Int. Stat., 5 p. 
LOETSCH F., HALLER K. E., 1966. Precision of estimate for management planning. Comptes rendus du Vle Congrès forestier mondial à Madrid (vol. 2), 2255-2264.

NYYSSÖNEN A., 1966. On the efficiency of some methods of forest survey. Comptes rendus du VIe Congrès forestier mondial à Madrid, 2473-2476.

NYYSSÖNEN A., ROIKO-JOKELA P., KILKKI P., 1971. Studies on improvement of the efficiency of systematic sampling in forest inventory. Acta forestalia fennica, 116, 26.

O'REGAN W. G., PALLEY M. N., 1965. A computer technique for study of forest sampling methods. For. Sci., 11 (1), 99-114.

PARDE J., 1956. Inventaires forestiers et méthodes statistiques. Rev. for. française, 8, 598-614.

PARDE J., 1961. Dendrométrie, Nancy, Edition de l'Ecole nationale des Eaux et Forêts, 350 p.

SMITH H. F., 1938. An empirical law describing heterogeneity in the yields of agricultural crop. J. Agr. Sci., 28, 1-23.

TARDIF G., 1965. Some considerations concerning the establishment of optimum plot size in forest survey. For. Chron., 41, (1), 93-102.

VILLENEUVE P., 1964. Calcul de l'erreur d'échantillonnage et détermination de l'aire optimale des parcelles-échantillons. Québec., Thèse de maîtrise. 